\title{
Design and Control Method of Switchable On- or Off-Ramp for Urban Highway
}

\author{
Yugang Liu, ${ }^{1,2,3,4}$ Hongbo Yi, ${ }^{1,2,3}$ Jiali Li, ${ }^{1,2,3,5}$ Bin Chen, ${ }^{4}$ and Hongtai Yang $\mathbb{D}^{1,2,3}$ \\ ${ }^{1}$ School of Transportation and Logistics, Southwest Jiaotong University, Chengdu 611756, China \\ ${ }^{2}$ National Engineering Laboratory of Integrated Transportation Big Data Application Technology, Southwest Jiaotong University, \\ Chengdu 611756, China \\ ${ }^{3}$ National United Engineering Laboratory of Integrated and Intelligent Transportation, Southwest Jiaotong University, \\ Chengdu 611756, China \\ ${ }_{4}^{4}$ Institute of Transportation Development Strategy \& Planning of Sichuan Province, Chengdu 610000, China \\ ${ }^{5}$ Department of Civil Engineering, Monash University, Melbourne, Australia
}

Correspondence should be addressed to Hongtai Yang; yanghongtai@swjtu.cn

Received 16 December 2019; Revised 10 June 2020; Accepted 8 July 2020; Published 1 September 2020

Academic Editor: Giuseppe Guido

Copyright (c) 2020 Yugang Liu et al. This is an open access article distributed under the Creative Commons Attribution License, which permits unrestricted use, distribution, and reproduction in any medium, provided the original work is properly cited.

\begin{abstract}
High volume of traffic is loaded on urban highway during peak hours as well as the ramp connecting main road and auxiliary road of highway. Once the demand exceeds the capacity of the ramp, congestion may happen on both main road and the auxiliary road. To deal with this problem, an new urban highway ramp, switchable ramp (SR) that can switch between on and off condition, is proposed in this paper. A macroscopic time-space discrete model is built in this paper to model this process by dividing the studied time period into short time periods and the studied road segment into small sections. The impact of the type of ramp on upstream and downstream traffic flow is analyzed. A segment of urban highway in Chengdu, China, is used as a case study. The traffic flow data in peak hours is collected and used. Mathematical analysis proves that the SR system proposed in this paper can reduce the total travel time (TTT) in the studied area. Results show that the SR system is more useful when the traffic volume is high.
\end{abstract}

\section{Introduction}

The urban highway is an important part of urban road network. It is usually composed of a main road where the vehicle can run at high speed and ramps connecting the main road and auxiliary road. The main road and the auxiliary road of the urban highway are always on the same level and parallel to each other. Vehicles get on and off the main road of highway through on-ramp and off-ramp.

As shown in Figure 1, when the traffic volume of onramp and off-ramp exceeds the queue storage space, which usually happens during peak hours, there will be vehicles queueing in front of the ramp. This results in lower speed of traffic in the exit or entrance lance [1]. There will be bottlenecks in these lanes. In order to avoid the occurrence of traffic congestion, studying ramp metering and new type of ramp is very important.
Related studies have proved that there are some measures to increase the capacity of ramps. Regarding onramp, the vehicles that enter the main road through the on-ramp will interweave with vehicles that are already on the main road. So, controlling the traffic entering main road through the on-ramp can improve the capacity of merging area $[2,3]$. Similarly, controlling the traffic entering auxiliary road through the off-ramp can improve the capacity of merging area on auxiliary road. However, the interaction between adjacent ramps along the expressway is inevitable. If the coordinated control of multiple ramps is considered, the capacity of ramps and highway could be further improved [4-11]. Considering the traffic demand on high fluctuates over time, if the traffic flow information is obtained and analyzed in real time, the best control method can also be applied in a realtime manner. Some researchers also find that real-time 


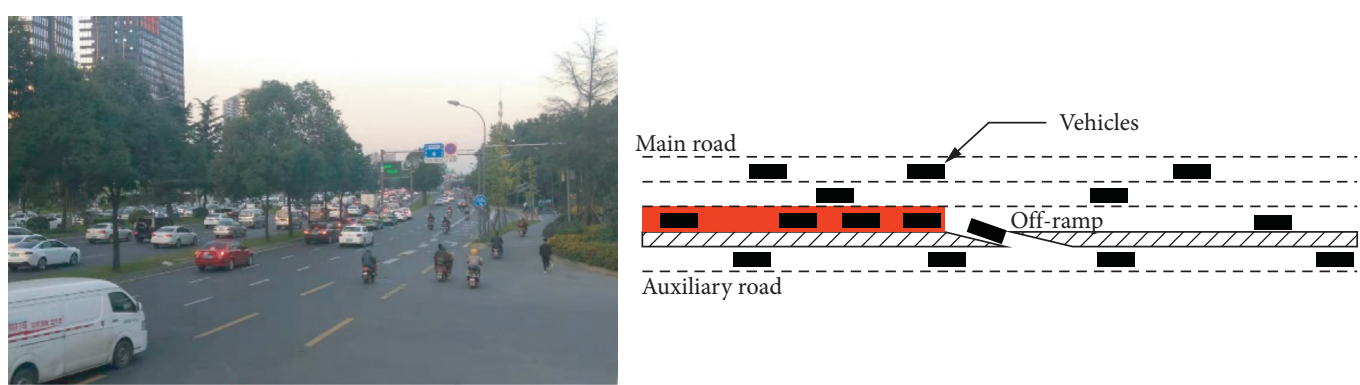

FIGURE 1: Queue of vehicles caused by traffic volume exceeding the queue storage space of ramp.

coordinated ramp metering strategy could better deal with the fluctuation of traffic flow [12-14]. It has also been found that the control method of variable speed limits (VSLs) along the main road has similar effect and ramp metering combined with VSL could achieve better results $[1,15,16]$. A control model for reducing congestion and emissions by combining ramp metering and route guidance was proposed by Pasquale et al. [17].

Ramp metering could reduce the highway congestion by limiting the number of vehicles passing through the ramp, which could also reduce the turbulence of platooned on-ramp vehicles and thus improve traffic safety. However, although ramp metering and other control methods can improve the traffic condition of main roads, these methods only consider the condition of the main road. When the demand to get on or to get off the highway varies, traditional ramp cannot meet varying demand well to balance the traffic pressure on the main road or auxiliary road.

Usually, existing ramps cannot meet the high traffic demand during peak hours. If we use fixed ramps, a pair of ramps (an on-ramp and an off-ramp) are needed. Besides, new fixed ramps may cause unnecessary weaving of vehicles during non-peak hours according to the shortcomings of the fixed ramp mentioned above. Therefore, it is necessary to design a type of switchable ramp (SR), which can switch condition according to the actual traffic condition. It can also be closed when it is not needed to avoid attracting extra demand. In terms of implementation, it is easy to let road users know the condition of the SR in real time using various methods such as variable message system (VMS) set upstream and map APP. The using of SR can meet the excessive traffic demand either on the main road or auxiliary road and reduce the possibility of platooned vehicles passing through the ramp as well. In addition, SR can also be used for other purposes, such as open during emergency for fire trucks or emergency vehicles.

This paper focuses on the design of SR and its optimal control method and discusses the security and operability issues of SR in the implementation process. The structure of this paper is as follows. Section 2 addresses the geometric design, operation, and implementation of SR. A macroscopic time-space discrete control model for SR is built in Section 3. A real case study is discussed in Section 4. Sections 5 and 6 present the conclusion and acknowledgments of this paper.

\section{Switchable Ramp on Urban Highway}

2.1. SR System. The SR can switch condition based on realtime traffic conditions. By using the cloud server, the optimal solution of the SR form can be determined based on realtime traffic data and a preset algorithm. We take two questions into consideration as follows when it comes to the implementation of SR.

First of all, we must inform the drivers of the real-time status of the SR. We recommend two ways of notification in this paper. One is VMS and the other is map APP. As long as drivers know the real-time condition of the SR, drivers could make better decision.

Secondly, the safety issues of SR in implementation are also considered. If the geometry of the SR is similar with that of a traditional fixed ramp, the SR will have similar safety performance. So, the safety issue should not be a concern.

The schematic diagram of the SR system is shown in Figure 2.

2.2. Geometry Design of SR. According to the reasons mentioned above, a type of switchable ramp is designed in this paper, which can switch its condition by simple physical transformation or traffic light. The form of SR could be one of these three: on-ramp, off-ramp, and closed. The choice of the condition depends on the real time traffic condition. The method to determine the condition of SR is explained in the Section 3. Generally speaking, it often turns into on- or offramp during peak hours and into closed condition when traffic demand is low. When the SR is in use, it can alleviate traffic congestion. When it is closed, it can reduce weaving traffic by reducing unnecessary lane changing. The design scheme of SR is introduced as follows.

As is shown in Figure 3, the arrow in each subgraph indicates the direction of the traffic flow. The upper side is the main road of urban highway, and the lower side is the auxiliary road of the highway. The shaded area represents the central isolation belt between main road of highway and auxiliary road. It often uses flower bed with vegetation of appropriate height. This would be the basis of realization of SR. The geometric design is described below.

The whole SR system are embedded into central isolation belt (the shaded area between auxiliary road and main road). The SR system is divided into three segments: area (a), area (b1), and area (b2). Every segment is different with area (a) 


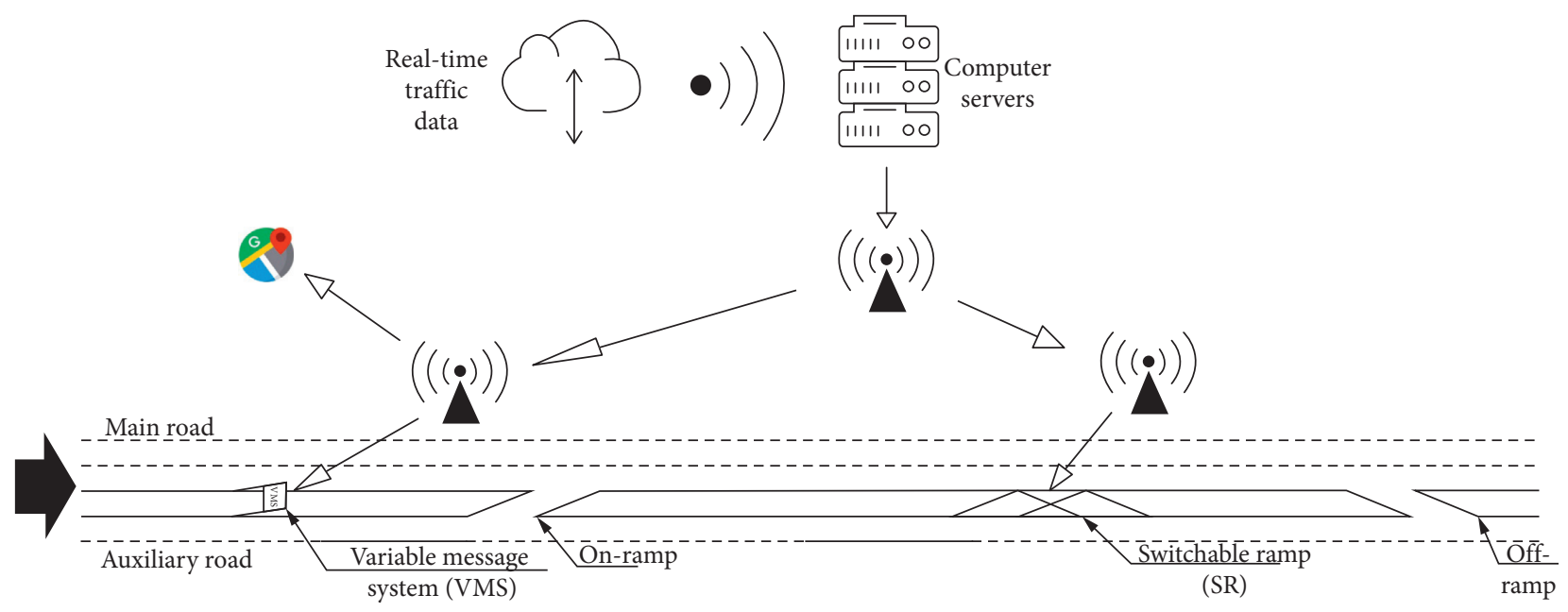

FIGURE 2: The geometric design of switchable ramp and two of its forms.

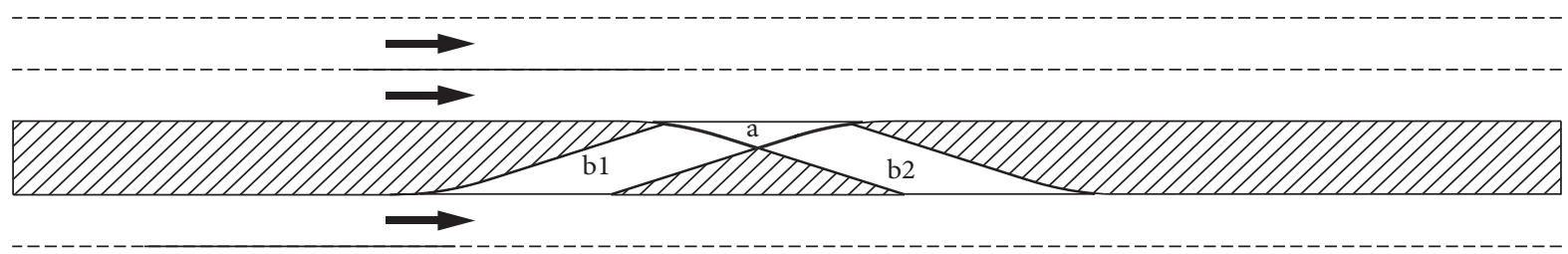

(a)

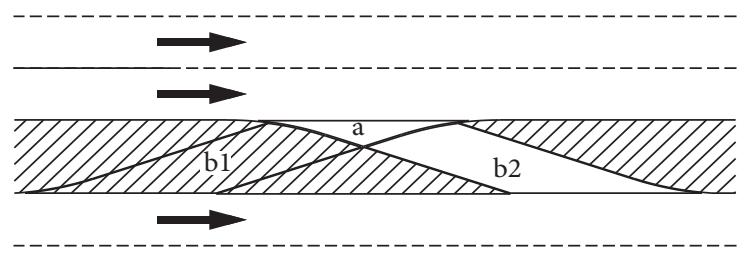

(b)

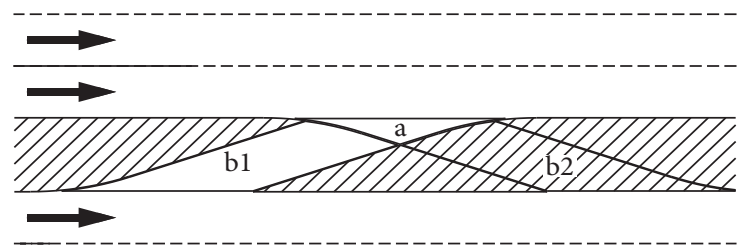

(c)

FIgURE 3: The geometric design of switchable ramp and two of its forms.

approximating a triangle and area (b1) and area (b2) approximating two trapezoids. It can be seen that area (b1) and area (b2) are axisymmetric. Area (a), area (b1), and area (b2) should be designed with curved edges to accommodate the vehicles' running streamline.

Each area is equipped with a board that can be raised and lowered. The upper limit of the rise of the board is an appropriate height at which vehicles cannot pass through. The lower limit at which the board can be lowered is at the same level as the surrounding road surface. If all boards rise to the highest point, the SR becomes closed and the vehicle cannot pass through. If the area (a) is lowered and then one of the areas (b1) and (b2) is lowered, an open ramp is formed. For example, when area (b1) is open and (b2) is blocked, it is an on-ramp.
In the following sections, we will introduce a control method for SR and verify its validity.

\section{Control Method}

3.1. Traffic Conditions on Main Roads under Normal Condition. According to the previous studies [18], the interaction of the three parameters to describe traffic state can be described as follows:

$$
\begin{aligned}
& v=V(\rho), \\
& q=\rho \cdot V(\rho),
\end{aligned}
$$

where $V(\rho)$ indicates that the parameter of velocity $v$ is a function determined by the density $\rho$. The velocity $v$ 
decreases with the increase of density $\rho$ and equals to zero when the density $\rho$ reaches the congestion density.

A macroscopic dispersed traffic flow model is used in model building in this paper, by describing the road network of the study area as a directed graph containing nodes and links. The traffic condition of a road segment is regarded as homogeneous. The road segments could be divided in a way to make sure that there are no ramps, big curves, or changes in the number of lanes in any segments. The location of the node should be the point where the ramp and the curves are located, and the point where the number of lanes changes. Similarly, we divide the entire study time into several time periods of length $\Delta T$. Therefore, the traffic state of the road segment $i$ of length $L_{i}$ within $\Delta T$ can be described as follows when $L_{i}$ and $\Delta T$ are small [1]:

$$
\begin{aligned}
v_{i, \Delta T} & =V\left(\rho_{i, \Delta T}\right), \\
Q_{i, \Delta T} & =\rho_{i, \Delta T} \cdot V\left(\rho_{i, \Delta T}\right) \cdot \Delta T,
\end{aligned}
$$

where $v_{i, \Delta T}$ represents the average vehicle speed of the road segment $i$ within time $\Delta T$ and $Q_{i, \Delta T}$ represents the traffic volume of the road segment within the time $\Delta T$ at segment $i$, which is expressed as the number of vehicles moving away from the downstream boundary of the road segment $i$ within $\Delta T$. Density $\rho_{i, \Delta T}$ is affected by road geometry, number of vehicles in the road segment, and lane change behavior of the vehicle $[19,20]$. The way that lane change behavior influences density $\rho_{i, \Delta T}$ will be introduced in Section 3.2.

\subsection{Influence of Lane Change Behavior on Traffic Condition of} Main Roads. When a vehicle changes lane, it will travel from the current lane to the target lane. During this period, the vehicle occupies those two lanes. It can be considered as having two cars traveling in line. As shown in Figure 4, the dotted rectangle in Figure 4(a) represents the actual lane change behavior, and the dotted rectangle in Figure 4(b) represents the equivalent case.

According to the analysis above, the equivalent density $\bar{\rho}$ on the segment $i$ in the time $\Delta T$ can be described as follows [21]:

$$
\bar{\rho}=\rho_{i, \Delta T}+\frac{N_{l_{-}} T_{l_{-}}}{L_{i} \Delta T},
$$

where $\rho_{L_{i}, \Delta T}$ represents the traffic flow density when there is no lane change behavior in the studied area, $\Delta T$ and $L_{i}$ represent the studied time period and space, respectively, $N_{l_{-} c}$ represents the number of lane changes in $\Delta T$ and $L_{i}$, and $T_{l_{-} c}$ represents the time needed for lane change behavior.

If we denote the traffic volume of the on-ramp as $q$ ' and the traffic volume of the main road as $q$, then the downstream traffic volume is $q+q$. The upstream or downstream boundaries of the studied area is set to satisfy the requirement that this area can contain all the lane change behavior caused by the ramp. The traffic volume of each lane in the upstream and downstream is $q / n$ and $\left(q+q^{\prime}\right) / n$, respectively, where $n$ is the number of lanes. In this condition, represented by Figure 5(a), the equivalent density $\bar{\rho}$ on the main road at downstream of the on-ramp is expressed as

$$
\begin{aligned}
\bar{\rho} & =\rho_{i, \Delta T}+\frac{q^{\prime} T_{l_{-} c}(n-1)}{2 L_{i}}, \\
N_{l_{-} c} & =q^{\prime} \frac{n-1}{2} \cdot \Delta T .
\end{aligned}
$$

For off-ramp, the lane change behavior happens on the main road on the upstream of the ramp, as shown in Figure 5(b). If we denote the traffic flow on the ramp as $q \prime$, the time for lane change is the same as case of on-ramp. The equivalent density $\bar{\rho}$ on the main road at upstream of the offramp is

$$
\bar{\rho}=\rho_{i, \Delta T}+\frac{q^{\prime} T_{l_{-}}(n-1)}{2 L_{i} \Delta T} .
$$

The lane change behavior increases the equivalent density of the segment. As mentioned earlier, increase in the number of lane changes reduces the traffic flow speed of the vehicle. The expression of function $V_{i, \Delta T}$ is

$$
V_{i, \Delta T}=\min \left\{V_{\text {lim }}, V_{f}^{i}\left(1-\bar{\rho}_{i, \Delta T} / \rho_{\text {con }}^{i}\right)\right\},
$$

where $\rho_{\text {con }}^{i}$ represents the congestion density, $V_{f}^{i}$ represents the free flow velocity, and $V_{\text {lim }}$ represents the speed limit of the road.

It can be seen from the function above that change of the equivalent density results in change of the average speed on the road segment, which leads to change of traveling time of all the vehicles. It is assumed that the traffic condition on the road segment $i$ is homogeneous during time $\Delta T$ when $\Delta T$ and $L_{i}$ are small enough. The number of vehicles in the section within $\Delta T$ can be recorded as $N_{i, \Delta T}$. The expected total traveling time (TTT) of those vehicles in the road segment $i$ during $\Delta T$ is

$$
\begin{aligned}
\operatorname{TTT}_{i, \Delta T} & =N_{i, \Delta T} \cdot L_{i} / V_{i, \Delta T}, \\
\operatorname{TTT}_{\Delta T} & =\sum_{i} T T T_{i, \Delta T} .
\end{aligned}
$$

where $\mathrm{TTT}_{i, \Delta T}$ represents the expected TTT of vehicles in road segment $i$, and $\mathrm{TTT}_{\Delta T}$ represents the overall expected TTT of vehicles of the studied area.

3.3. Nodes Model between Road Segments. In this paper, the road to be studied is divided into several segments and nodes connecting these segments for modeling purposes. It should be noted that the node acts as a virtual point connecting the upstream and downstream road segments and does not have spatial attributes. So that nodes cannot store vehicles like road segments. Therefore, for the node $N_{i}$, at any time,

$$
\begin{aligned}
& q_{N_{i}}^{\text {in }}=\sum_{i \in I_{N_{i}}} q_{i}^{\text {out }}, \\
& q_{N_{i}}^{\text {out }}=\sum_{i \in O_{N_{i}}} q_{i}^{\text {in }},
\end{aligned}
$$

where the parameters $q_{N_{i}}^{\text {in }}$ and $q_{N_{i}}^{\text {out }}$ represent the inflow and outflow of the node $N_{i}$, respectively, $I_{N_{i}}$ represents a set of road segments on the upstream side of node $N_{i}$, and 


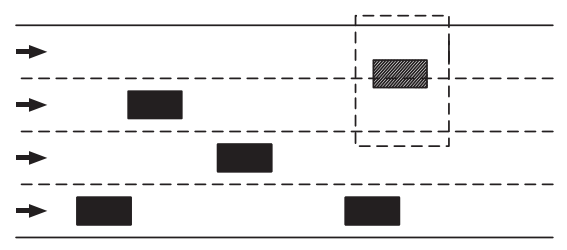

(a)

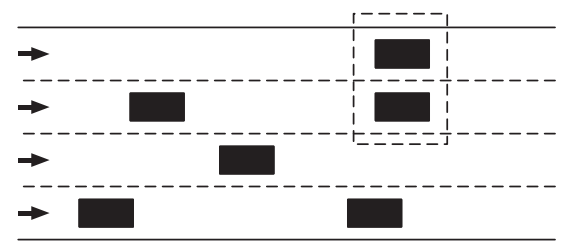

(b)

Figure 4: Lane change behavior and its equivalent case.

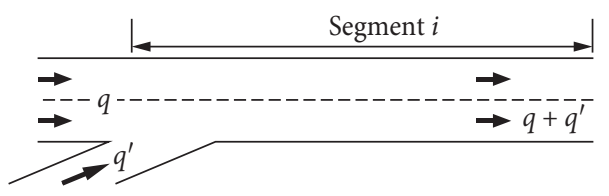

(a)

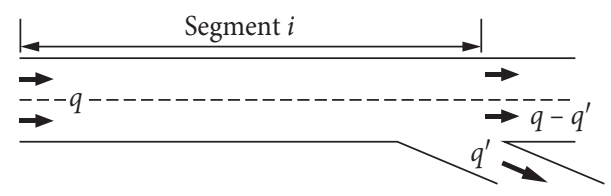

(b)

Figure 5: Analysis of traffic flow of on-ramp and off-ramp.

similarly, $O_{N_{i}}$ represents the set of road segments on the downstream side of node $N_{i}$. The relationship between the segments and the node is shown in Figure 6.

The following three principles should be followed when selecting the location of the node:

(i) The traffic conditions on the upstream and downstream side of the ramp are often different. So, the point where the ramp is located should be selected as the node.

(ii) When the distance between two adjacent ramps is too long, in order to ensure that the length of each segment is small enough, appropriate number of points should be selected along the road as nodes.

(iii) When road geometry changes, these points should be selected as nodes.

A node can only be one of the three types: normal node (without ramp), on-ramp, or off-ramp. The type of node $N_{i}$ can be denoted as $S_{N_{i}}$, with values of 1, 0 , and -1 indicating on-ramp, normal, and off-ramp nodes.

3.4. Model of Time Dimension. The traffic condition of each road segments is changing constantly over time. In this section, we discuss the relationship between traffic flow in the $(k+1) t h \Delta T$ and traffic flow in the previous time period $k$ th $\Delta T$. The equivalent density recursion function between two time periods is as follows:

$$
\begin{gathered}
\bar{\rho}_{i, \Delta T}^{k+1}=\rho_{i, \Delta T}^{k+1}+\rho_{i, \Delta T}^{\prime k+1}, \\
\rho_{i, \Delta T}^{\prime k+1}=\frac{N_{l_{-c}}^{k+1} T_{l_{-c}}}{L_{i} \Delta T}, \\
\rho_{i, \Delta T}^{k+1}=\rho_{i, \Delta T}^{k}+\Delta T \frac{\left[q_{i, \Delta T}^{\text {in }}(k)-q_{i, \Delta T}^{\text {out }}(k)\right]}{L_{i}},
\end{gathered}
$$

$$
q_{N_{i}}^{\text {out }}(k+1)=\min \left\{\sum_{j \in O_{N_{i}}}\left[Q_{j}^{c}-Q_{j}(k)\right] \sum_{j \in O_{N_{i}}} q_{j}^{\text {in }}(k)\right\} .
$$

where $Q_{j}^{c}$ represents the maximum number of vehicles that can be accommodated by road segment $j$ and $Q_{j}(k)$ represents the vehicle that is already on the road segment $j$ in the $k t h \Delta T$ time. According to the equations above, the traffic condition at $(k+1) t h \Delta T$ can be calculated by the following equations:

$$
\begin{gathered}
V_{i, \Delta T}(k+1)=\min \left\{V_{\lim }^{i}, V_{f}^{i}\left(\frac{1-\bar{\rho}_{i, \Delta T}^{k+1}}{\rho_{c}^{i}}\right)\right\}, \\
q_{i, \Delta T}^{\text {in }}(k+1) \frac{q_{i, \Delta T}^{\text {in }}(k)}{\sum_{i \in O_{N_{i}}} q_{i, \Delta T}^{\text {in }}(k)}=q_{N_{i-1}, \Delta T}^{\text {out }}(k+1), \\
\sum_{i \in I_{N_{i}}} q_{i, \Delta T}^{\text {out }}(k+1)=q_{N_{i}}^{\text {in }}(k+1)=q_{N_{i}}^{\text {out }}(k+1), \\
q_{i, \Delta T}^{\text {out }}(k+1)=\frac{q_{i, \Delta T}^{\text {out }}(k)}{\sum_{i \in I_{N_{i}}} q_{i, \Delta T}^{\text {out }}(k)} \sum_{i \in I_{N_{i}}} q_{i, \Delta T}^{\text {out }}(k+1) .
\end{gathered}
$$

Equations (9) to (16) can be summarized as follows:

$$
x(k+1)=f\left[x(k), s_{N_{i}}(k+1)\right],
$$

where $x(k)$ represents the state of traffic flow during the time period $k t h \Delta T$ and $s_{N_{i}}(k+1)$ represents the type of nodes during the time period $(k+1) t h \Delta T$.

3.5. Optimization Objective. During peak hours or in an emergency, the capacity of fixed ramps may not be able to meet the high traffic demand. In some time periods, the traffic demand from the main road to the auxiliary road is greater, and sometimes it is the contrary. In these two cases, the condition of SR could be adjusted according to the actual 


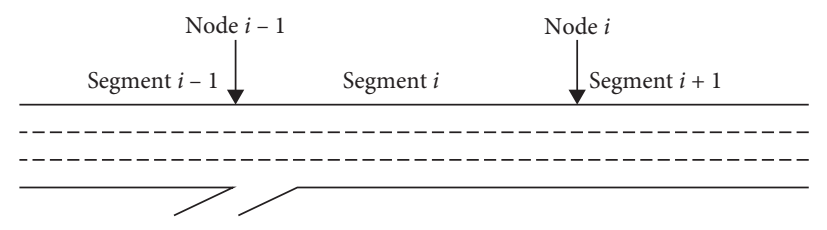

FIgURE 6: Relationship between segments and nodes.

traffic state. When the traffic demand is not high, fixed ramps can meet all the requirements. Thus, the SR should be turned into the closed condition to reduce unnecessary weaving of vehicles.

In this paper, we set minimizing TTT as the optimization objective. The form of SR in $(k+1) t h \Delta T$ is determined by the traffic state of the time period $k t h \Delta T$ :

$$
\begin{aligned}
& \min \operatorname{TTT}(k+1)=\sum_{i} \operatorname{TTT}_{i, \Delta T}^{k+1}, \\
& \text { s.t. }\left\{\begin{array}{l}
x(k+1)=f\left[x(k), s_{N_{i}}(k+1)\right], \\
\left|S_{N_{i}}(k+1)\right|=0 \text { or } 1, \\
k=1,2, \ldots, n,
\end{array}\right.
\end{aligned}
$$

When the traffic demand is high and the capacity of existing ramp cannot meet the demand, there will be a large number of vehicles accumulating in the upstream segment of the ramp. According to equation (6), when the traffic flow density of a road segment reaches congestion density, the traffic flow speed of the road segment will be equal to zero. In this case, opening the SR could allow vehicles to pass through to reduce congestion. The probability that the density of weaving segments reaches congestion density is also reduced. In the Section 4 , a case study is performed to prove this point.

\section{Case Study}

4.1. Data of the Case. A four-lane main road and two-lane auxiliary road of about 2.03 kilometers, located in Chengdu, China, is used for our case study. It is presented in Figure 7. The road segment is between Supo-flyover (103.9906E, $30.6763 N)$, on the upstream of the section, and Yangxiflyover $(104.0047 E, 30.7037 N)$, on the downstream of the section. We divided the studied area into 20 small segments, with the length of each segment being about 100 meters. There are only one fixed on-ramp (node $N_{5}$ ) and one fixed off-ramp (node $N_{13}$ ) along the road. Distance between these two ramps is about 800 meters. Traffic data of Monday, July 8, 2019, were used, from 5:00 PM to 5:30 PM. Traffic volume on the main road and auxiliary road is shown as Tables 1 and 2. A hypothetical SR (node $N_{9}$ ) is set at the midpoint between the on-ramp and off-ramp.

The average velocity of traffic in initial condition at starting point on the upstream of the main road is 43.808 kilometers per hour and that of the auxiliary road is 36.768 kilometers per hour. The average density of traffic at starting point of main road and auxiliary road is $19.325 \%$ and $32.263 \%$, respectively. According to the study of Greenshields [22]; the free flow velocities of the main and auxiliary roads are around 67 kilometers per hour and 49 kilometers per hour, respectively. The speed limit of the main road is 80 kilometers per hour and that of the auxiliary road is 60 kilometers per hour. The time needed for a vehicle to change lanes is set as 10.5 seconds [20].

4.2. Optimization Effect When Adding an $S R$ at Node $\mathbf{N}_{9}$. In this section, we analyze the change in traffic condition after adding an SR under two types of traffic demands. The results are as follows.

Figure 8(a) indicates the traffic condition of the studied area without SR. The $x$ axis represents the road segment $i$ (from 1 to 20), and the $y$ axis represents the time $k$ (from 1 to 170). Step size $\Delta T$ is selected as 10 s. Different colors represent different TTTs of the segment $i$ in $k t h \Delta T$, i.e., $\mathrm{TTT}_{i, \Delta T}(k)$. Figure $\left.8(\mathrm{~b}) 1\right)$ represents the curve of overall TTT $\left(\operatorname{TTT}_{\Delta T}(k)\right)$ over time in the no SR case. When the traffic demand in the studied area increases by $5 \%$, the traffic condition without SR is shown in Figure 8(a)). The coordinate system in Figure $8(\mathrm{a})$ ) is the same as the coordinate system in Figure 8(a)). Figure 8(b)) shows the overall TTT curve over time when there is no SR.

The results of both two cases above when SR is used are shown in other subfigures. When the traffic demand is set as the current traffic demand, results are shown in Figures 8(c) and $8(\mathrm{~d})$. When the traffic demand increases by $5 \%$, results are shown in Figures 8(c) and 8(d). Moreover, Figure 8(e) shows the curve of $s_{N_{9}}\left(N_{9}\right.$ is the node with SR in this case). As defined before, 1,0 , and -1 represent on-ramp, normal, and off-ramp, respectively.

The mathematical analysis proves that the SR system proposed in this paper can reduce TTT contrast to no SR case. Under the current traffic flow condition, the TTT can be reduced from 148.107 hours to 142.024 hours, with a reduction of $4.107 \%$. When traffic demand increases by $5 \%$, the TTT of highway system can be reduced from 157.289 hours to 150.262 hours, with a reduction of $4.468 \%$.

The reduction mentioned above is the reduction in the TTT over the study period. But SR is not open all the time. If we only compare the reduction of TTT during the opening period of SR, the reduction rate of TTT under the surveyed traffic flow is $6.416 \%$, and the reduction rate of TTT when traffic flow increases by $5 \%$ is $9.526 \%$. The increase in traffic flow leads to more significant effects.

4.3. Comparison of Effects of $S R$ and Traditional Ramp. The analysis above demonstrates that the SR proposed in this paper is effective in reducing the overall TTT at the surveyed traffic flow condition and when the volume increases by $5 \%$. In order to verify that adding a SR has better effect than 


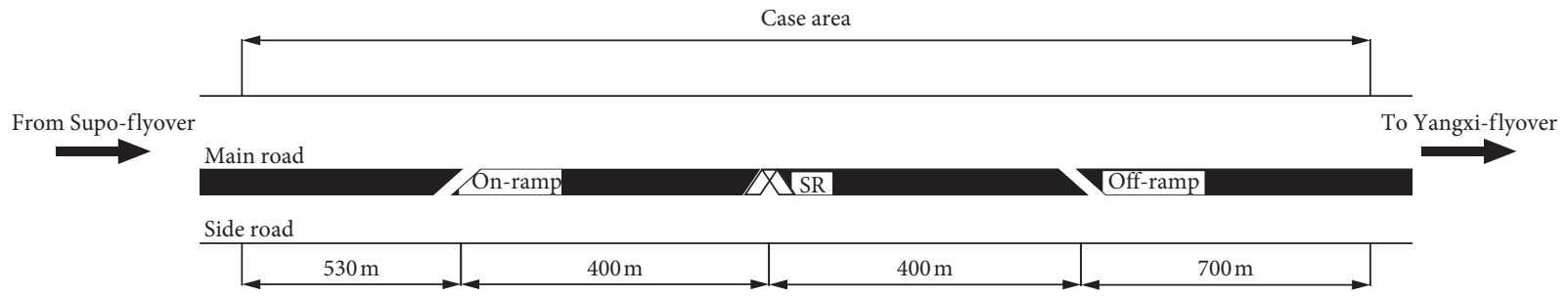

Figure 7: Case study area.

TABle 1: Traffic volume of main road.

\begin{tabular}{|c|c|c|c|c|c|c|c|c|c|c|}
\hline \multirow{2}{*}{$\begin{array}{l}\text { Time index } \mathbf{k} \\
1 \text { to } 10\end{array}$} & \multicolumn{10}{|c|}{ Flow rate $(\mathrm{pcu} / \mathrm{h} /$ lane $)$} \\
\hline & 1548 & 1260 & 855 & 900 & 828 & 1278 & 1800 & 1440 & 918 & 630 \\
\hline 11 to 20 & 756 & 585 & 720 & 810 & 846 & 630 & 900 & 1458 & 360 & 360 \\
\hline 21 to 30 & 360 & 360 & 360 & 360 & 360 & 360 & 360 & 360 & 360 & 360 \\
\hline 31 to 40 & 360 & 360 & 360 & 558 & 360 & 630 & 738 & 990 & 675 & 1188 \\
\hline 41 to 50 & 1980 & 2295 & 2070 & 2187 & 1980 & 2268 & 1818 & 540 & 720 & 1440 \\
\hline 51 to 60 & 1440 & 900 & 1683 & 1080 & 1440 & 1800 & 1170 & 1188 & 1350 & 1080 \\
\hline 61 to 70 & 2340 & 2133 & 1845 & 1890 & 1836 & 1800 & 1530 & 1638 & 1809 & 2475 \\
\hline 71 to 80 & 1278 & 1782 & 1188 & 2124 & 1926 & 1710 & 990 & 1116 & 2340 & 2178 \\
\hline 81 to 90 & 2043 & 1656 & 2079 & 1710 & 1701 & 2124 & 2205 & 1440 & 630 & 540 \\
\hline 91 to 100 & 1440 & 1350 & 1098 & 810 & 1080 & 810 & 1170 & 1413 & 1080 & 1260 \\
\hline 101 to 110 & 1170 & 1755 & 630 & 270 & 1521 & 1314 & 1530 & 855 & 360 & 648 \\
\hline 111 to 120 & 828 & 1278 & 1296 & 1008 & 1530 & 630 & 693 & 1296 & 1080 & 450 \\
\hline 121 to 130 & 1026 & 990 & 1548 & 1530 & 810 & 1530 & 1278 & 630 & 1125 & 1260 \\
\hline 131 to 140 & 810 & 1620 & 1548 & 1440 & 1530 & 1080 & 900 & 900 & 936 & 1350 \\
\hline 141 to 150 & 1530 & 1683 & 1368 & 1530 & 1188 & 720 & 1098 & 918 & 990 & 360 \\
\hline 151 to 160 & 1440 & 720 & 630 & 720 & 2358 & 900 & 630 & 558 & 630 & 1566 \\
\hline 161 to 170 & 1251 & 720 & 1215 & 720 & 738 & 378 & 270 & 360 & 3816 & 180 \\
\hline
\end{tabular}

Table 2: Traffic volume of auxiliary road.

\begin{tabular}{lcccccccccc}
\hline Time index $\mathbf{k}$ & \multicolumn{7}{c}{ Flow rate (pcu/h/lane) } \\
\hline 1 to 10 & 1566 & 360 & 360 & 360 & 360 & 900 & 1080 & 720 & 1080 & 360 \\
11 to 20 & 360 & 360 & 360 & 360 & 360 & 360 & 360 & 540 & 360 & 360 \\
21 to 30 & 360 & 360 & 360 & 360 & 360 & 360 & 360 & 360 & 360 \\
31 to 40 & 360 & 360 & 360 & 360 & 360 & 360 & 360 & 360 & 360 & 720 \\
41 to 50 & 900 & 2520 & 1800 & 1170 & 1656 & 720 & 972 & 540 & 360 & 360 \\
51 to 60 & 360 & 720 & 1260 & 1062 & 1980 & 1080 & 1260 & 900 & 1260 & 900 \\
61 to 70 & 1980 & 1692 & 1872 & 1440 & 1080 & 1620 & 1080 & 900 & 1440 \\
71 to 80 & 990 & 900 & 720 & 1080 & 1260 & 720 & 540 & 846 & 1260 & 1260 \\
81 to 90 & 1440 & 900 & 1116 & 1260 & 900 & 1656 & 1080 & 1080 & 540 \\
91 to 100 & 1530 & 1080 & 990 & 360 & 1116 & 360 & 1296 & 540 & 810 \\
101 to 110 & 720 & 900 & 720 & 450 & 360 & 576 & 360 & 576 & 720 \\
111 to 120 & 576 & 360 & 360 & 360 & 360 & 360 & 360 & 720 & 720 \\
121 to 130 & 360 & 576 & 360 & 360 & 360 & 360 & 540 & 360 & 360 \\
131 to 140 & 540 & 360 & 540 & 720 & 360 & 720 & 360 & 360 & 540 \\
141 to 150 & 360 & 360 & 360 & 360 & 540 & 540 & 360 & 360 & 540 \\
151 to 160 & 576 & 540 & 720 & 540 & 720 & 540 & 540 & 1800 & 810 \\
161 to 170 & 540 & 360 & 756 & 720 & 900 & 540 & 540 & 1152 & 1152 \\
\hline
\end{tabular}



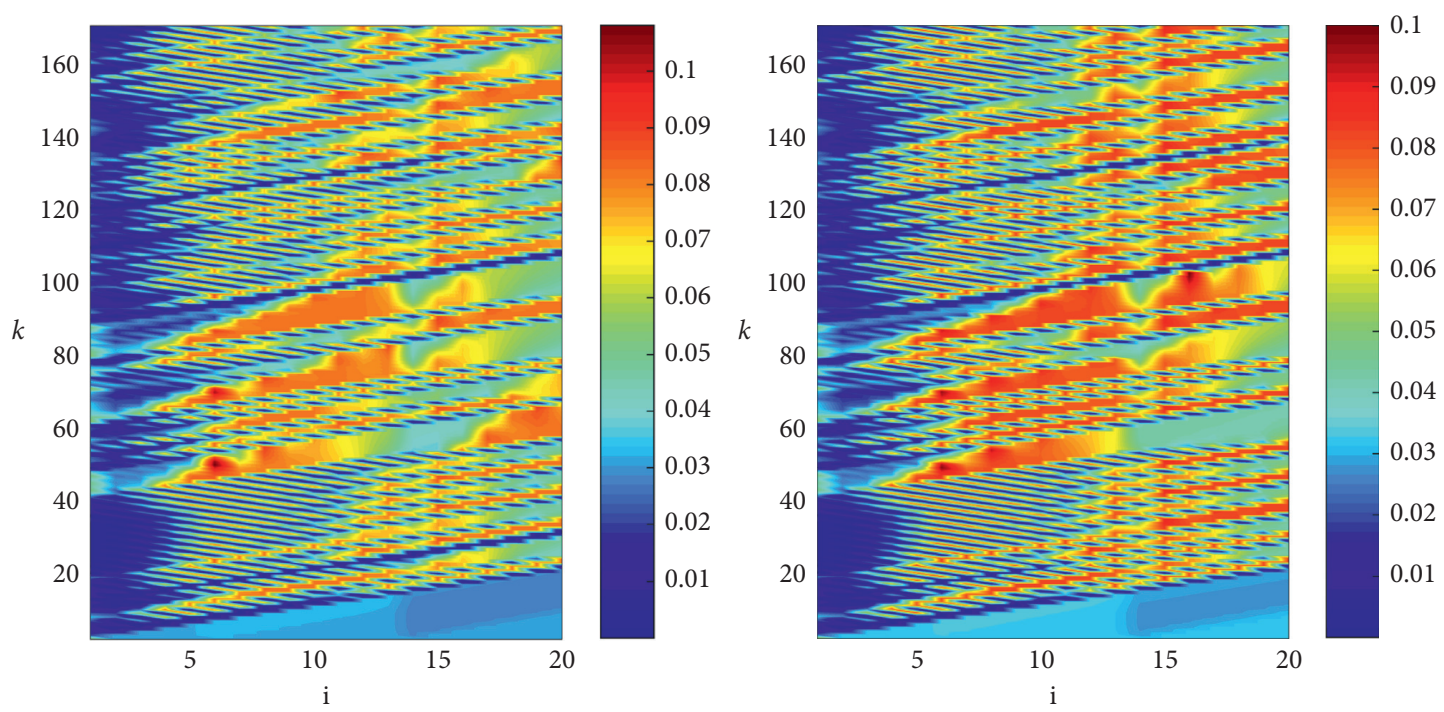

(a)
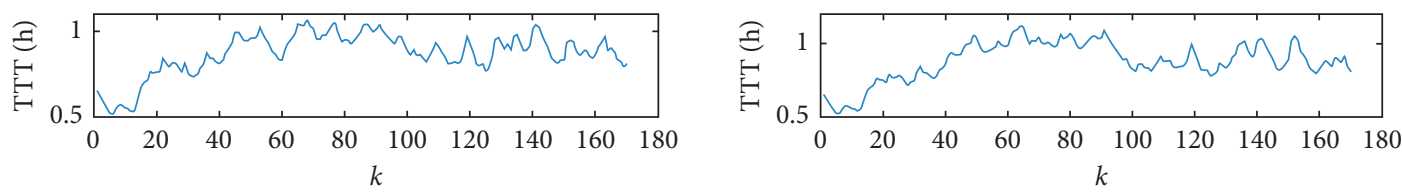

(b)
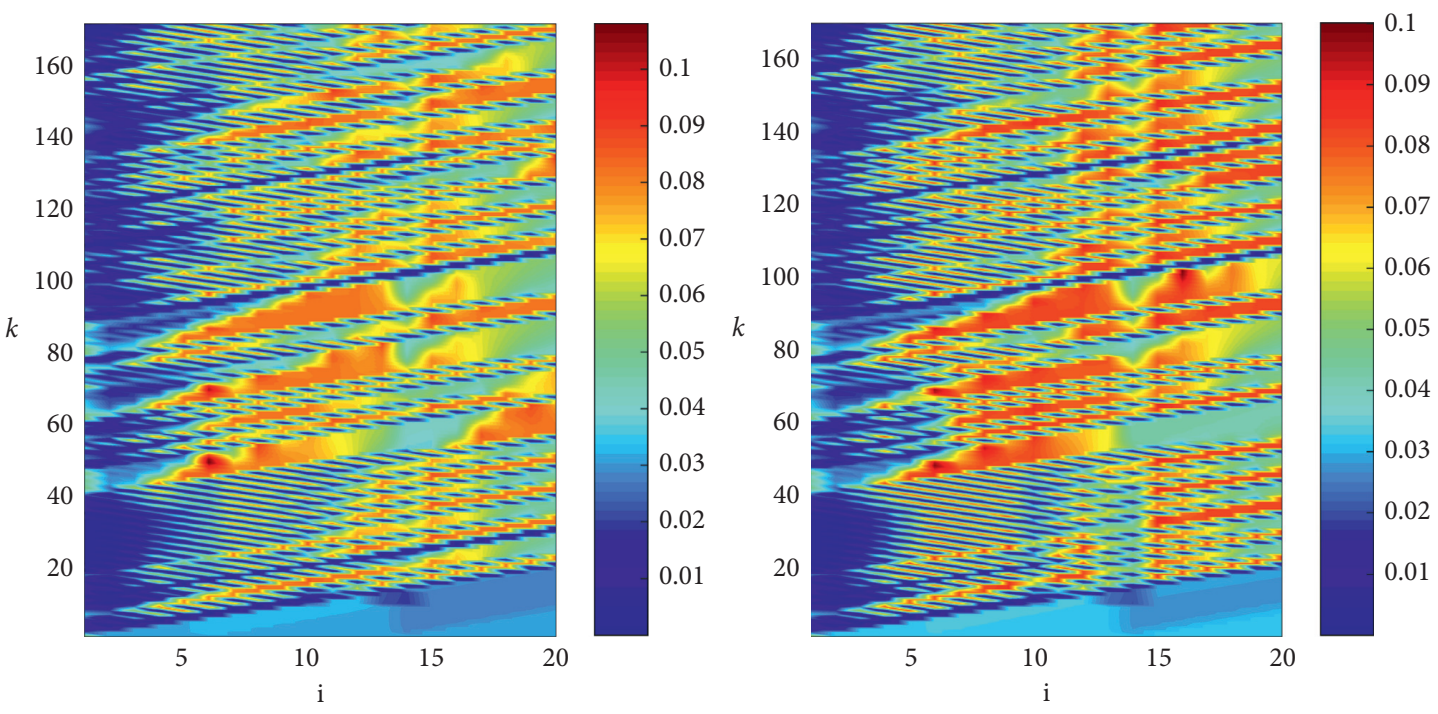

(c)
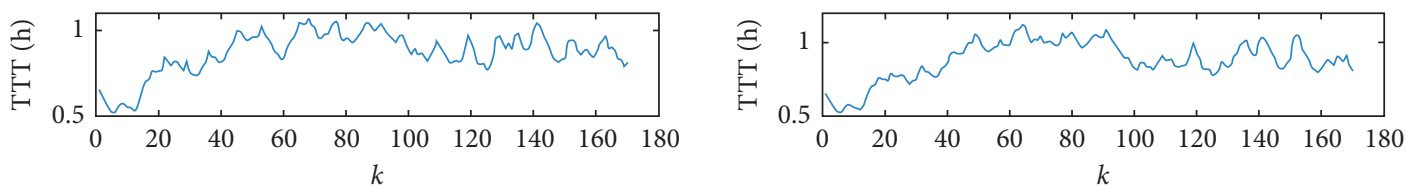

(d)
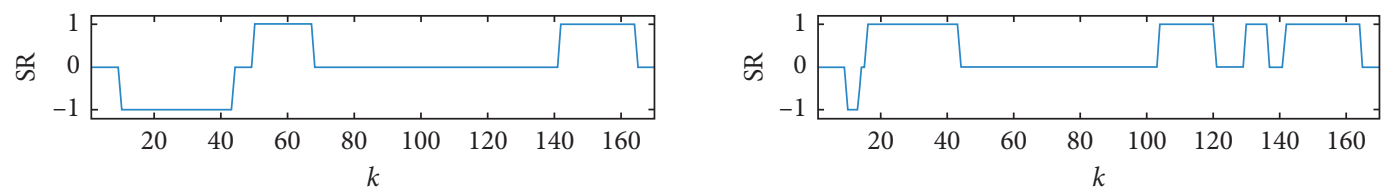

(e)

Figure 8: Results of case study. 
TABLE 3: TTT (hours) under different traffic flows.

\begin{tabular}{|c|c|c|c|c|c|c|c|}
\hline Multiple of original flow & 0.90 & 0.93 & 0.935 & 0.94 & 0.95 & 1.00 & 1.05 \\
\hline Scenario 1 & 131.082 & 136.694 & 137.546 & 138.398 & 140.828 & 148.107 & 157.289 \\
\hline Scenario 2 & 131.082 & 136.694 & 137.281 & 137.724 & 140.105 & 142.024 & 150.262 \\
\hline Scenario 3 & 131.853 & 136.868 & 137.414 & 138.208 & 140.514 & 146.837 & 155.382 \\
\hline Scenario 4 & 132.144 & 136.970 & 137.335 & 138.061 & 140.467 & 145.066 & 153.776 \\
\hline
\end{tabular}

adding a traditional on-ramp or off-ramp, we analyze TTT under different traffic flow conditions. The results are shown in Table 3. Four scenarios are considered:

\section{Scenario 1: no change \\ Scenario 2: add an SR at $N_{9}$ \\ Scenario 3: add an on-ramp at $N_{9}$ \\ Scenario 4: add an off-ramp at $N_{9}$}

The first row in Table 3 indicates the input traffic flow, represented by the number multiplying the original traffic flow.

The gray cells in each column indicate the lowest TTT in the column, which are the optimal cases. We found that the TTT of Scenario 1 is the shortest when the input flow is less than or equal to 0.93 times the surveyed flow. Adding a ramp in these conditions will increase TTT. Thus, when the traffic flow is less than or equal to 0.93 times, the SR should be in the closed form, which is equivalent to no ramp. When the input traffic flow is greater than or equal to 0.94 times the surveyed traffic flow, no matter which type of ramp is added, the TTT will decrease. When SR is added, the TTT is the lowest.

In order to find out the critical point where SR can improve the traffic condition, we studied in more detail about the traffic flow between 0.93 and 0.94 times the surveyed flow and found that the critical point is 0.935 times the surveyed traffic flow in this case.

\section{Conclusion}

This paper proposes a type of SR for urban highway that can be easily implemented. It can be used in the case that the main road and auxiliary road of urban highway are on the same level. A spatial-temporal discrete model is proposed to minimize the TTT of all the vehicles in the studied area by adjusting the condition of SR. The idea of the model is to divide the studied road segment into several short sections and the studied time period into small intervals to calculate the travel time of the vehicles in each section and time interval. The impact of the condition of SR on the traffic flow of adjacent main road and auxiliary road is analyzed.

At the end of the paper, an expressway segment of about 2 kilometers in Chengdu, China is used to demonstrate the control method. By applying the mathematical analysis, the adoption of SR could reduce the TTT of vehicles traveling in the segment. When the traffic demand is higher, the percent of reduction of TTT is also higher, indicating the SR is more effective. The critical threshold value is also determined. When the traffic demand is higher than the critical threshold value, the SR should be turned on.
There are also several limitations of this paper. First of all, this paper only considers the impact of SR on traffic condition of surrounding area when a new SR is added. In the following research, we will study the coordinated control of multiple SRs in the overall urban road network. Another limitation of this study is that the decision of turning the SR to on, off, or closed condition depends largely on the traffic volume on the main and auxiliary road. The method to determine the condition of SR is somehow complicated. Thus, there is no simple guideline to show when to turn the SR condition to on, off, or closed. Thirdly, the decision of adding a ramp or switching ramp between on and off condition depends on a lot of factors such as human factors. Those factors are not considered in this paper. Future studies could focus on how to consider these factors when designing SR. However, the setup of SR depends on existing technologies and could be regarded as a combination of on and off-ramp with metering. This combination is not considered before and a control method is proposed for this type of random. This type of ramp may not be implemented immediately because a lot of factors such as travellers' reaction are not considered. But, we believe it is a valuable exploration and this type of ramp may be implemented in the future.

\section{Data Availability}

The data used to support the finding of this study are available within the article.

\section{Conflicts of Interest}

The authors declare that they have no conflicts of interest.

\section{Acknowledgments}

This study was funded by the National Natural Science Foundation of China (grant numbers 51774241 and 71704145), Sichuan Youth Science and Technology Innovation Research Team Project (grant number 2020JDTD0027), Humanity and Social Science Foundation of Ministry of Education of China (grant number 18YJCZH138), and China Postdoctoral Science Foundation.

\section{References}

[1] R. C. Carlson, I. Papamichail, M. Papageorgiou, and A. Messmer, "Optimal motorway traffic flow control involving variable speed limits and ramp metering," Transportation Science, vol. 44, no. 2, pp. 238-253, 2010.

[2] Y. Kan, Y. Wang, M. Papageorgiou, and I. Papamichail, "Local ramp metering with distant downstream bottlenecks: A 
comparative study," Transportation Research Part C: Emerging Technologies, vol. 62, no. 1, pp. 149-170, 2016.

[3] M. J. Cassidy and J. Rudjanakanoknad, "Increasing the capacity of an isolated merge by metering its on-ramp," Transportation Research Part B: Methodological, vol. 39, no. 10, pp. 896-913, 2005.

[4] O. J. Chen, A. F. Hotz, and M. E. Ben-Akiva, "Development and evaluation of a dynamic ramp metering control model," IFAC Proceedings Volumes, vol. 30, no. 8, pp. 1089-1095, 1997.

[5] A. Kotsialos and M. Papageorgiou, "Efficiency and equity properties of freeway network-wide ramp metering with AMOC," Transportation Research Part C: Emerging Technologies, vol. 12, no. 6, pp. 401-420, 2004.

[6] G. Gomes and R. Horowitz, "Optimal freeway ramp metering using the asymmetric cell transmission model," Transportation Research Part C, vol. 14, no. 4, pp. 244-262, 2006.

[7] I. Papamichail, A. Kotsialos, I. Margonis, and M. Papageorgiou, "Coordinated ramp metering for freeway networks-a modelpredictive hierarchical control approach," Transportation Research Part C: Emerging Technologies, vol. 18, no. 3, pp. 311-331, 2010.

[8] J. Martin, S. Martin, M. Payer, A. M. Bayen et al., "Creating complex congestion patterns via multi-objective optimal freeway traffic control with application to cyber-security," Transportation Research Part B: Methodological, vol. 91, no. 9, pp. 366-382, 2016.

[9] M. Schmitt, C. Ramesh, and J. Lygeros, "Sufficient optimality conditions for distributed, non-predictive ramp metering in the monotonic cell transmission model," Transportation Research Part B: Methodological, vol. 105, no. 11, pp. 401-422, 2017.

[10] M. Lygeros, I. Karafyllis, and M. Papageorgiou, "Local and coordinated ramp metering within the unifying framework of an adaptive control scheme," Transportation Research Part A: Policy and Practice, vol. 128, no. 10, pp. 89-113, 2019.

[11] Y. Han, M. Ramezani., A. Hegyi, Y. Yuan, and S. Hoogendoorn, "Hierarchical ramp metering in freeways: An aggregated modeling and control approach," Transportation Research Part C: Emerging Technologies, vol. 110, no. 1, pp. 1-19, 2020.

[12] M. Papageorgiou, J.-M. Blosseville, and H. Haj-Salem, "Modelling and real-time control of traffic flow on the southern part of Boulevard Périphérique in Paris-Part I: Modelling," Transportation Research Part A: General, vol. 24, no. 5, pp. 345-359, 1990.

[13] M. Papageorgiou, J. M. Blosseville, and H. Haj-Salem, "Modelling and real-time control of traffic flow on the southern part of Boulevard Périphérique in Paris-Part II: coordinated on-ramp metering," Transportation Research Part A: General, vol. 24, no. 5, pp. 361-370, 1990 b.

[14] M. Papageorgiou, I. Papamichail, A. D. Spiliopoulou, and A. F. Lentzakis, "Real-time merging traffic control with applications to toll plaza and work zone management," Transportation Research Part C: Emerging Technologies, vol. 16, no. 5, pp. 535-553, 2008.

[15] A. Alessandri, A. Di Febbraro, A. Ferrara, and E. Punta, "Optimal control of freeways via speed signalling and ramp metering," Control Engineering Practice, vol. 6, no. 6, pp. 771-780, 1998.

[16] K. Kampitaki, Integrated Control of Traffic Flow Using Ramp Metering and Variable Speed Limits, M.S. thesis, Technical University of Crete, Chania, Greece, 2008.

[17] C. Pasquale, S. Sacone, S. Siri, and B. De Schutter, "A multiclass model-based control scheme for reducing congestion and emissions in freeway networks by combining ramp metering and route guidance," Transportation Research Part C: Emerging Technologies, vol. 80, no. 7, pp. 384-408, 2017.

[18] G. F. Newell, "A simplified car-following theory: a lower order model," Transportation Research Part B: Methodological, vol. 36, no. 3, pp. 195-205, 2002.

[19] G.-L. Chang and Y.-M. Kao, "An empirical investigation of macroscopic lane-changing characteristics on uncongested multilane freeways," Transportation Research Part A: General, vol. 25, no. 6, pp. 375-389, 1991.

[20] W.-L. Jin, "A multi-commodity Lighthill-Whitham-Richards model of lane-changing traffic flow," Transportation Research Part B: Methodological, vol. 57, no. 11, pp. 361-377, 2013.

[21] L.C. Edie, "Discussion on traffic stream measurements and definitions," in Proceedings of the Second International Symposium on the Theory of Road Traffic Flow, pp. 139-154, London, UK, 1963.

[22] B. D Greenshields, "A study in highway capacity," Highway Research Board Proceedings, vol. 14, pp. 448-477, 1935. 